\title{
Metadata versus Full-Text: Tracking Users' Electronic Theses and Dissertations (ETDs) Seeking Behavior
}

Daniel Gelaw Alemneh and Mark Edward Phillips University of North Texas, Denton TX 76201, USA Daniel.Alemneh@unt.edu and Mark.Phillips@unt.edu

\section{iConference 2018}

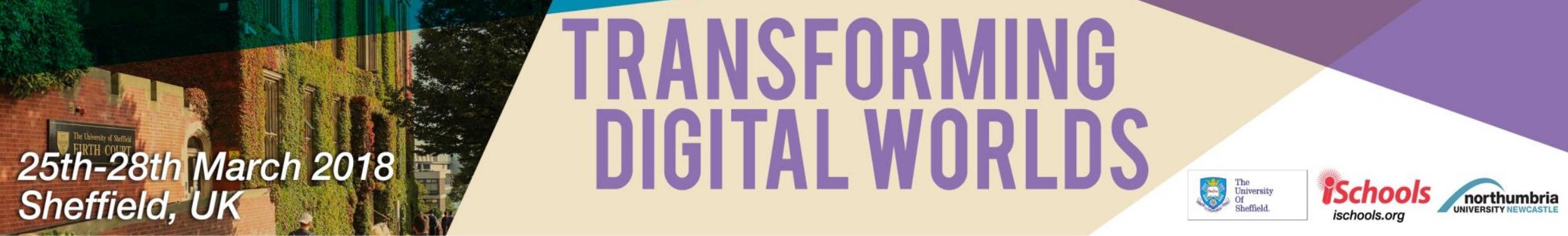




\section{Outline}

- Background

- ETD at UNT

- Usage Statistics

- Characteristics of ETDs

- ETDs Usage

- ETDs Discovery via Metadata Vs. Full-Text

- Methods, Analysis, and Findings

- Summary

- Future works 


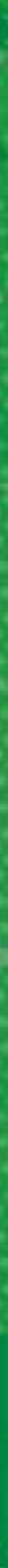




\section{ETD at UNT}

- The University of North Texas (UNT) began accepting theses and dissertations in electronic format in 1999.

- UNT is one of the early adopters of what was to become the ETD movement in higher education

- One of the first three American universities to require ETDs for graduation. 


\section{UNT \& ETD}

- At UNT, we have a combination of both born-digital ETDs and digitized analog these and dissertations.

- The ETD collection consists of more than 18,000 items and more than 2 mil-lion pages or files.

- More than 12,000 analog theses and dissertations (from 1936 to 1999) were digitized

- More than 6,000 born digital ETDs after fall-1999.

- The UNT Libraries play an active role in facilitating access to UNT's ETDs

- Integrate Value added services into the ETDs

- Multiple formats (PDF, JPG, )

- Integrate Related contents (Datasets, videos, audios e.g. recitals) 


\section{Overview}

$$
\begin{array}{r}
18,900 \text { Total ltems } \\
2,314,099 \text { Total liles } \\
9,363,367 \text { Total U lses }
\end{array}
$$

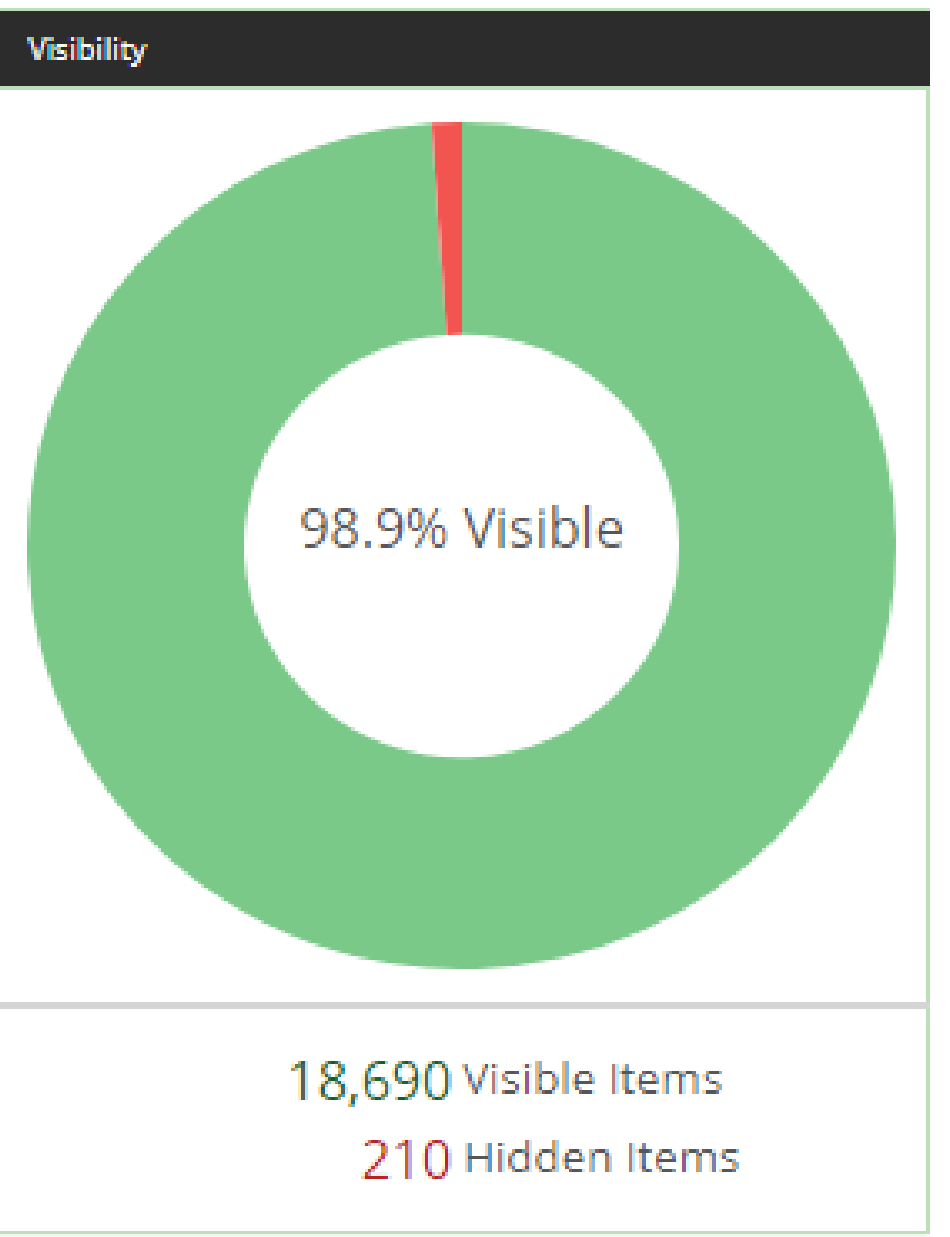

A green light to greatness: 


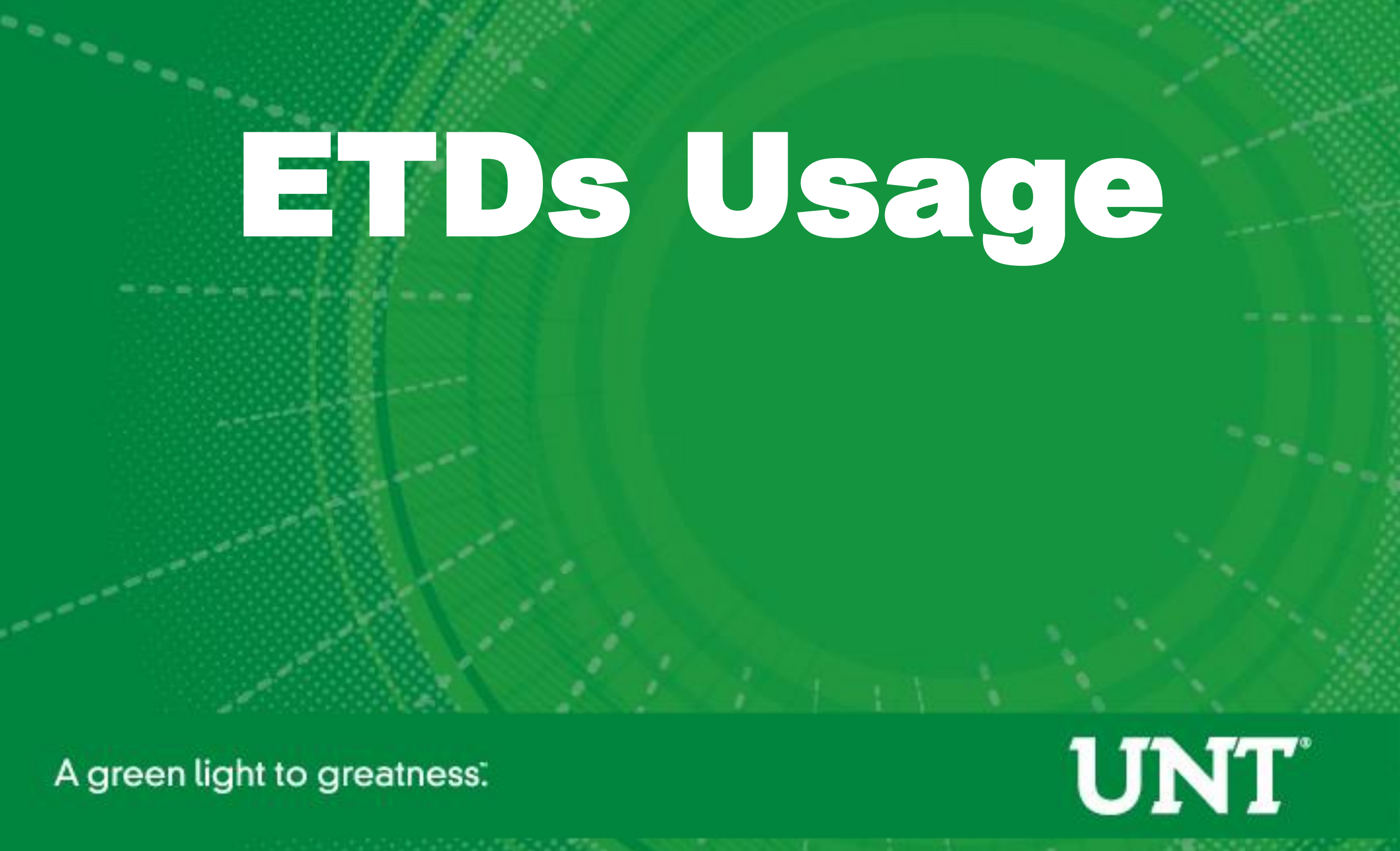




\section{ETDs Usage Statistics}

- UNT tracks the use of its ETD, as well as the use of other digital collections hosted by UNT

Libraries.

- ETD Collection is heavily used, with daily use level ranging between 2,000 and over 6,000 uses from $200+$ countries. 


\section{Usage}

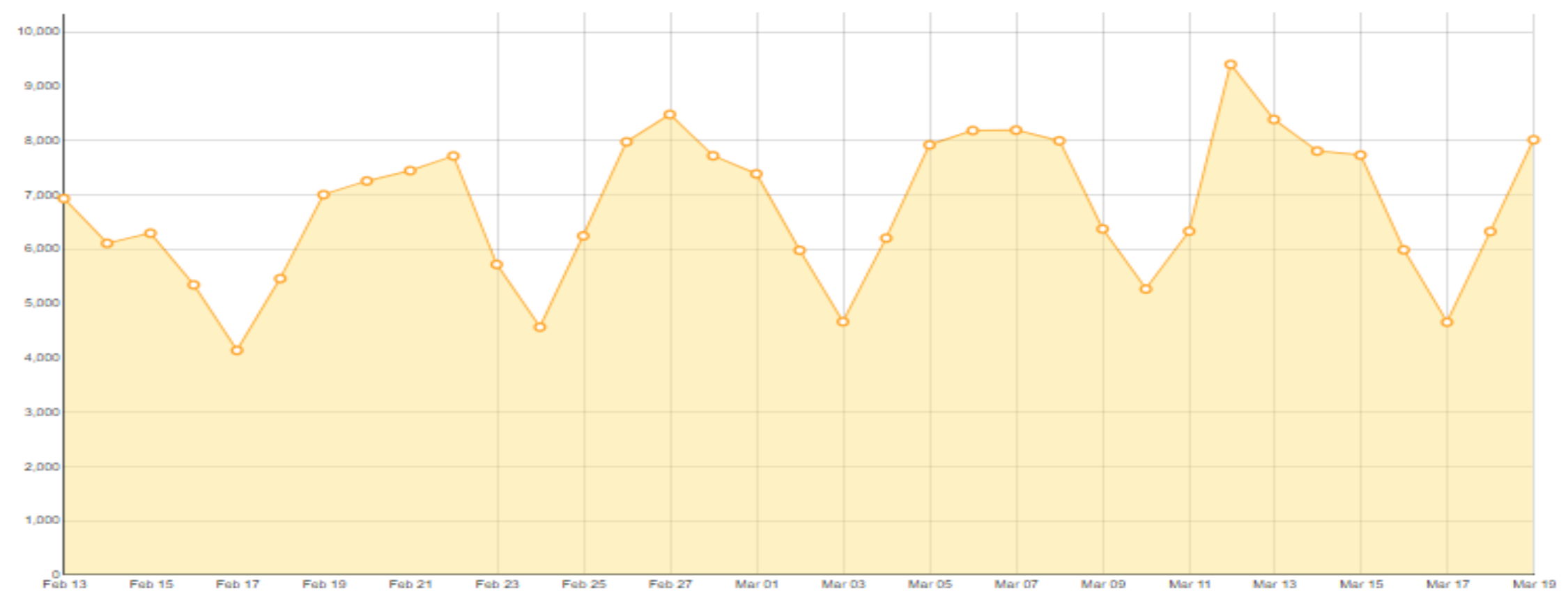

Recent usage

\section{Usage by Month/Year}

\begin{tabular}{|c|c|c|c|c|c|c|c|c|c|c|c|}
\hline Year & January & February & March & April & May & June & July & August & September & October & Noveml \\
\hline 2018 & 151,193 & 175,866 & 132,644 & & & & & & & & \\
\hline 2017 & 128,437 & 118,873 & 151,646 & 150,332 & 146,061 & 108,400 & 101,730 & 101,445 & 142,058 & 162,700 & 165,889 \\
\hline 2016 & 138,837 & 164,215 & 176,924 & 167,523 & 141,502 & 130,470 & 105,206 & 119,758 & 154,088 & 190,269 & 159,359 \\
\hline 2015 & 134,963 & 116,623 & 129,656 & 148,227 & 134,242 & 103,647 & 101,629 & 102,067 & 134,457 & 158,863 & 166,169 \\
\hline 2014 & 91,109 & 97,216 & 108,147 & 115,545 & 107,376 & 100,094 & 256,453 & 104,855 & 100,832 & 112,120 & 112,932 \\
\hline 2013 & 79,828 & 85,073 & 91,746 & 93,782 & 91,401 & 84,386 & 110,618 & 128,683 & 127,176 & 111,411 & 112,834 \\
\hline 2012 & 52,627 & 64,297 & 62,338 & 62,427 & 58,394 & 48,135 & 50,941 & 53,428 & 60,254 & 80,274 & 80,226 \\
\hline 2011 & 13,374 & 15,350 & 26,757 & 35,767 & 45,195 & 44,404 & 36,092 & 29,477 & 37,370 & 45,170 & 47,258 \\
\hline 2010 & 9,641 & 18,573 & 23,620 & 24,678 & 17,369 & 13,500 & 11,500 & 10,598 & 14,524 & 13,330 & 14,216 \\
\hline
\end{tabular}




\section{Visits from 215 Countries}

http://digital.library.unt.edu/explore/collections/UNTETD/browse/

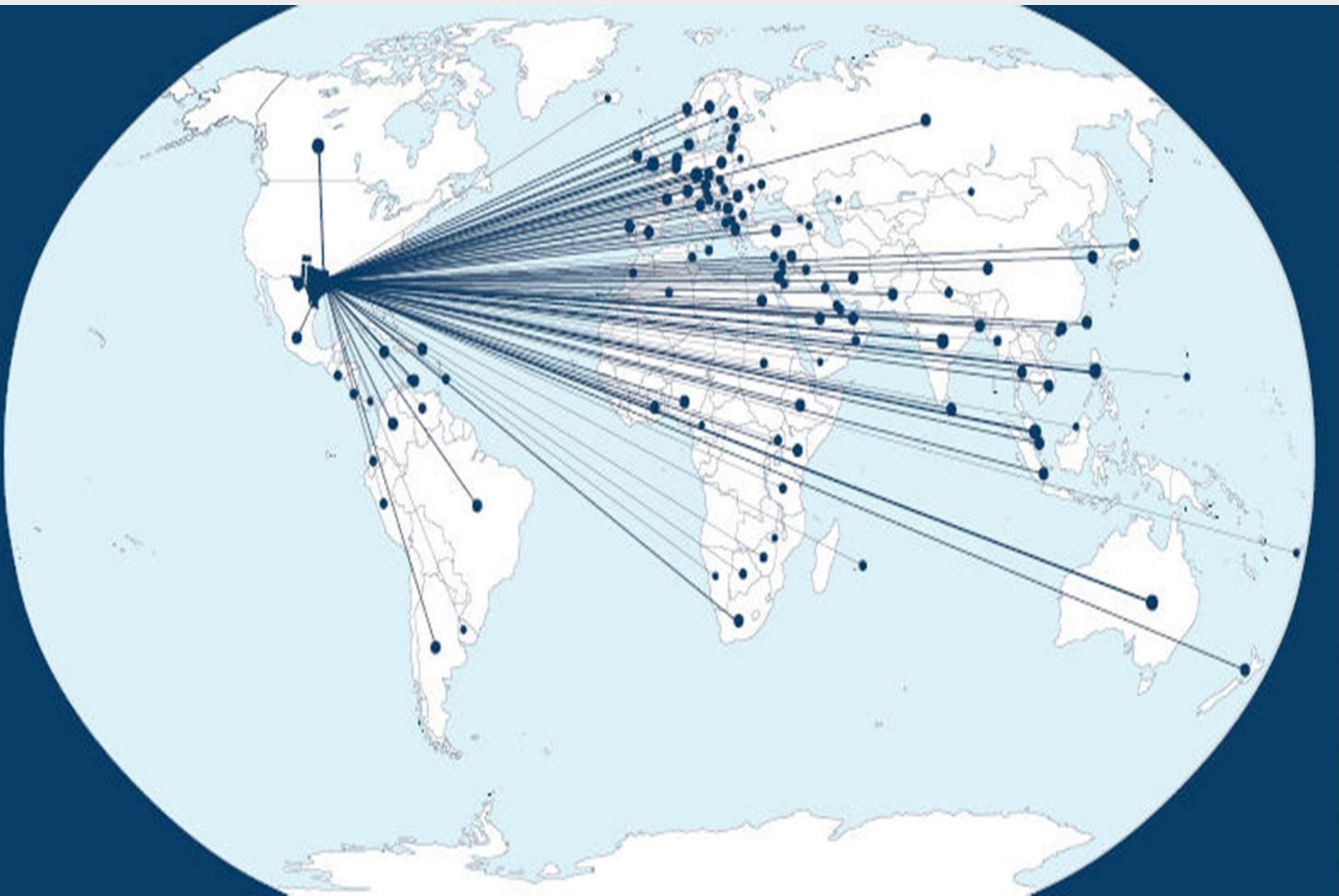




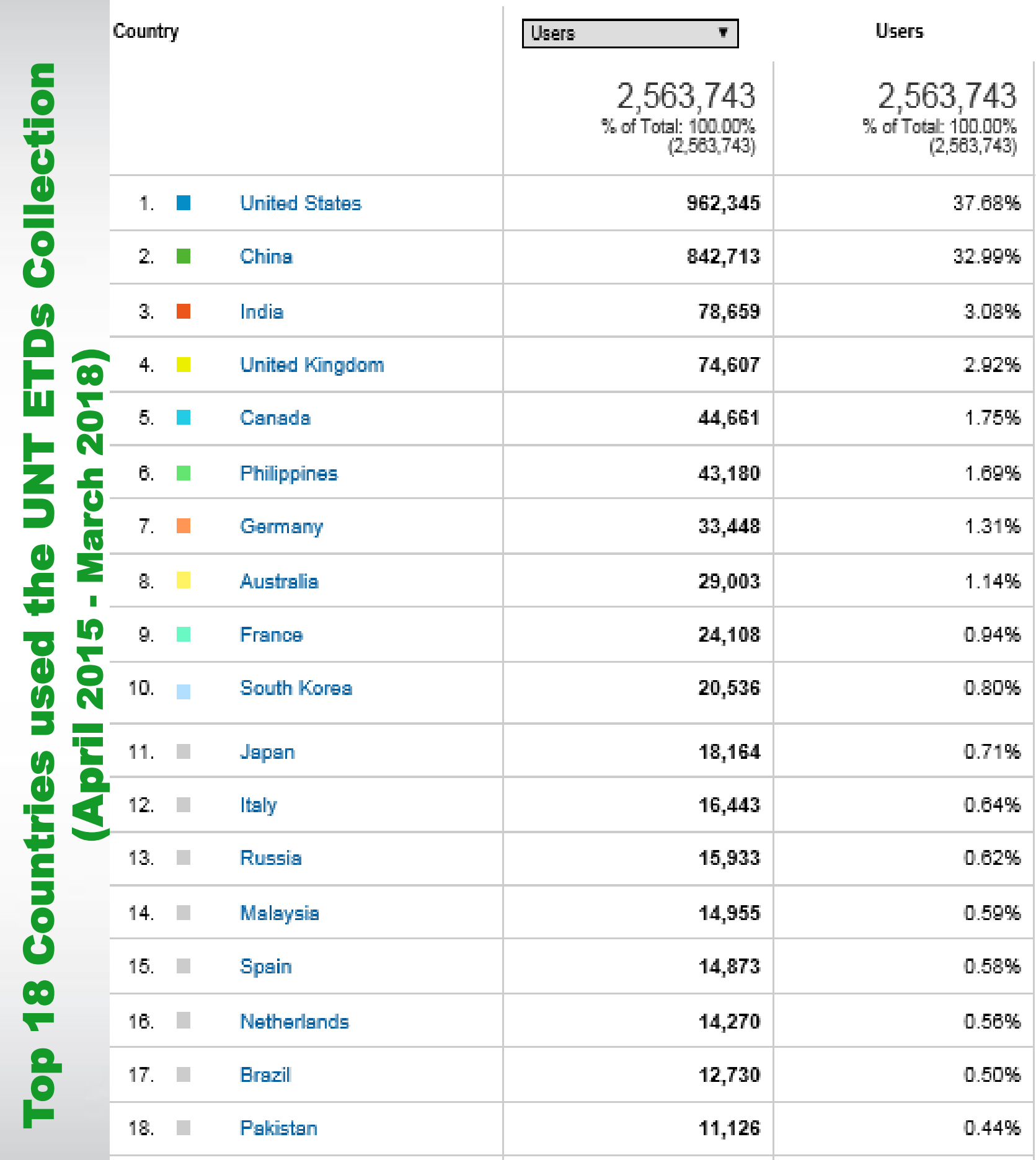




\begin{tabular}{|c|c|c|c|c|c|c|}
\hline Browser & Users & $\begin{array}{l}\text { New } \\
\text { Users }\end{array}$ & Sessions & $\begin{array}{c}\text { Bounce } \\
\text { Rate }\end{array}$ & $\begin{array}{l}\text { Pages / } \\
\text { Session }\end{array}$ & $\begin{array}{l}\text { Avg. Session } \\
\text { Duration }\end{array}$ \\
\hline Chrome & 192,863 & 184,101 & 220,364 & $57.56 \%$ & 2.52 & 0:02:08 \\
\hline Safari & 64,930 & 62,742 & 73,431 & $63.97 \%$ & 2.5 & $0: 01: 42$ \\
\hline Firefox & 41,733 & 39,474 & 45,728 & $58.92 \%$ & 2.55 & $0: 02: 17$ \\
\hline $\begin{array}{l}\text { Internet } \\
\text { Explorer }\end{array}$ & 34,314 & 33,006 & 36,668 & $64.75 \%$ & 2.13 & $0: 01: 41$ \\
\hline Edge & 13,911 & 13,507 & 15,920 & $58.70 \%$ & 2.95 & $0: 02: 42$ \\
\hline Opera Mini & 6,183 & 6,135 & 6,718 & $69.38 \%$ & 1.66 & 0:01:03 \\
\hline (not set) & 4,887 & 4,887 & 2,687 & $98.25 \%$ & 1 & 0:00:01 \\
\hline UC Browser & 3,329 & 3,234 & 3,662 & $73.38 \%$ & 1.53 & $0: 00: 58$ \\
\hline Opera & 2,402 & 2,318 & 2,949 & $60.09 \%$ & 2.16 & 0:02:05 \\
\hline Android Browser & 1,558 & 1,510 & 1,629 & $78.08 \%$ & 1.34 & 0:01:10 \\
\hline $\begin{array}{l}\text { Android } \\
\text { Webview }\end{array}$ & 1,367 & 1,355 & 1,546 & $68.43 \%$ & 2.46 & $0: 02: 40$ \\
\hline Safari (in-app) & 1,284 & 1,248 & 1,391 & $60.68 \%$ & 2.96 & 0:01:10 \\
\hline $\begin{array}{l}\text { Samsung } \\
\text { Internet }\end{array}$ & 939 & 916 & 1,082 & $57.12 \%$ & 2.22 & $0: 01: 46$ \\
\hline Amazon Silk & 713 & 701 & 761 & $71.88 \%$ & 1.45 & $0: 02: 30$ \\
\hline
\end{tabular}




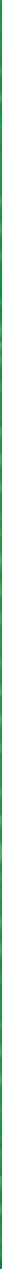

A green light to greatness: 


\section{ETDs Case Study}

- To get a better sense of users discovery of digital resources, we decided to assesse and see:

- Whether users were arriving at our digital resources from searches that were answered by an items descriptive metadata or by parts of the full-text of the item.

- This study analyzed access to UNTs ETD Collection from two sides:

- Searches that were answered by an items descriptive metadata

- Users request met by parts of the full-text of the item. 


\section{Methodology}

$>$ For use analysis, we used Web server logs from the application server that provides access to the UNT Digital Library.

-Before we extract the specific requests for ETDs in the UNT Digital Library, the data was obtained from a server log that contained 172 Million lines of requests

-The log files were limited to discoveries of items in the UNT ETDs collection

$>$ At the time this research was conducted, (Occurred between May 4, 2014 and January 24, 2015):

- There were 11,873 unique ETDs available with metadata 


\section{Methodology}

$>$ The original raw dataset contained $172,115,682$ lines during that timeframe, in the standard Extended Log File Format.

$>$ The resulting (two-column) intermediary dataset contained 84,837 item-query pairs

$>$ Further limitations removed:

$>\bullet$ Requests made by known robots,

$>\bullet$ Requests without known search queries,

$>$ Following further normalization, the dataset contained 43,420 unique query results; 


\section{Statistics for the Number of Tokens Per Query}

\begin{tabular}{|c|c|c|c|c|c|c|}
\hline N & Min. & Median & Max & Sum & Mean & Studdev \\
\hline 43420 & 1 & 2.5 & 31 & 104102 & 2.40 & 1.59 \\
\hline
\end{tabular}

- Queries varied in length and they were analyzed as individual words (or tokens) rather than phrases.

- This allowed for partial matches in a given field,

- The distribution of tokens across queries ranged from 1 to 31 tokens 


\section{Example Dataset Entries for Three Search Queries}

\begin{tabular}{|c|c|c|c|}
\hline Dataset Field & Example 1 & Example 2 & Example 3 \\
\hline Item & metadc129697 & metadc 146510 & metadc 155618 \\
\hline Query & susan cheal & human trafficking & article writing \\
\hline Query Tokens & 2 & 2 & 2 \\
\hline PageText & $0 \%$ & $100 \%$ & $100 \%$ \\
\hline Metadata & $100 \%$ & $100 \%$ & $50 \%$ \\
\hline Title & $0 \%$ & $100 \%$ & $50 \%$ \\
\hline Subject & $0 \%$ & $100 \%$ & $0 \%$ \\
\hline Agent & $100 \%$ & $0 \%$ & $0 \%$ \\
\hline Description & $0 \%$ & $100 \%$ & $0 \%$ \\
\hline
\end{tabular}


Record Discoveries on Matches in Metadata and Full-Text ( $N=43420)$

\begin{tabular}{|l|c|c|}
\hline \multicolumn{1}{|c|}{$\begin{array}{c}\text { Matches found in: Bottom of } \\
\text { Form }\end{array}$} & $\begin{array}{c}\text { Total No. of } \\
\text { Queries Found: \% }\end{array}$ & $\%$ \\
\hline Any part of query in full text & 41,519 & $95.6 \%$ \\
\hline Any part of query in metadata & 33,779 & $77.8 \%$ \\
\hline Both any metadata and full text & 32,056 & $73.8 \%$ \\
\hline $100 \%$ of query in full text (all tokens) & 36,318 & $83.6 \%$ \\
\hline $\begin{array}{l}\text { Queries ONLY in full text (but not in } \\
\text { metadata) }\end{array}$ & 9463 & $21.8 \%$ \\
\hline $\begin{array}{l}100 \% \text { of query in metadata (all tokens) } \\
\text { Queries ONLY in metadata (but not in } \\
\text { full text) }\end{array}$ & 29661 & $68.3 \%$ \\
\hline
\end{tabular}

\section{A green light to greatness:}




\section{Percentage of Queries Found:}

Queries ONLY in metadata (not in full text)

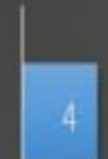

$100 \%$ of query in metadata

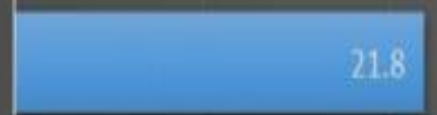

$100 \%$ of query in full text

Both any metadata and full text

Any part of query in metadata

\section{Anvert quer}

Any part of query in full text

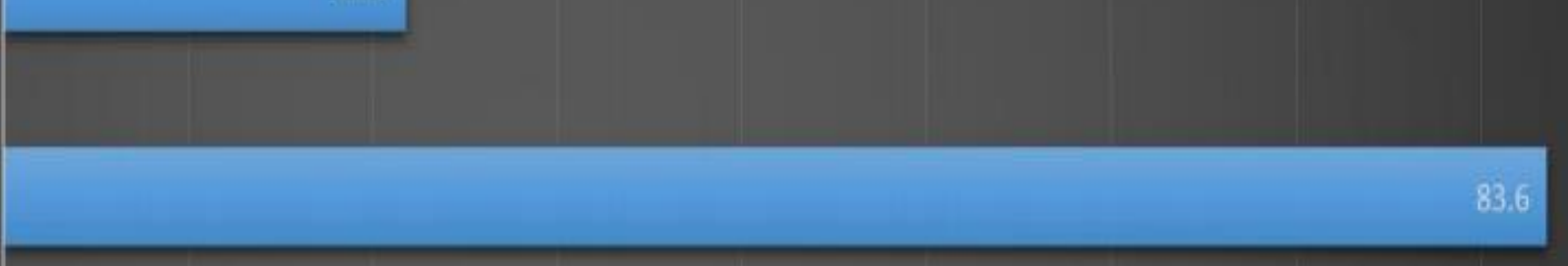




\section{Percentage of Matches Queries Found:}

\section{Queries ONLY in full}

text (22\%)

\section{Queries ONLY in}

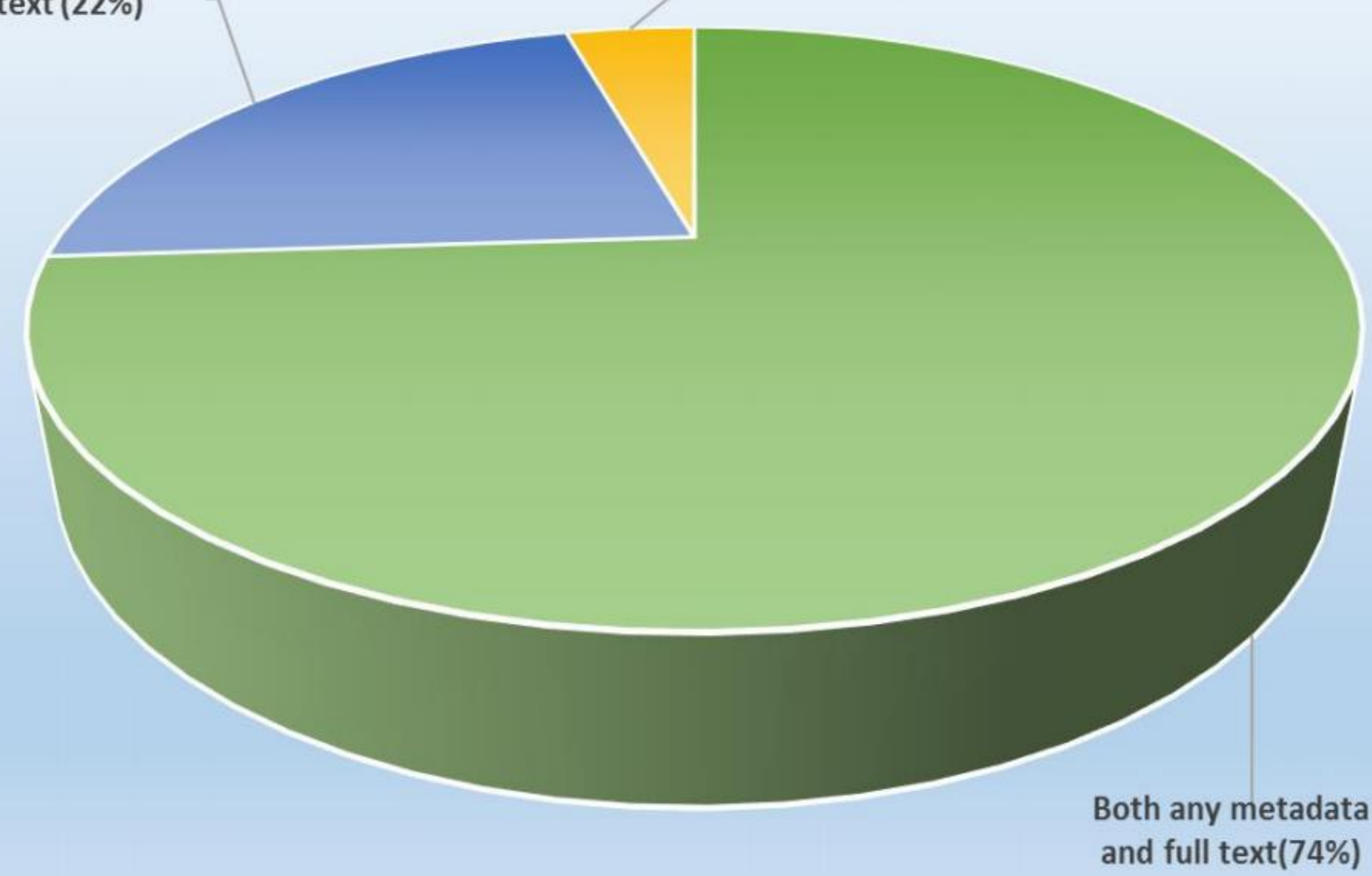


RECORD DISCOVERIES PER FIELD BASED ON PERCENTAGE OF QUERY PRESENT IN FIELD. (N=43420)

At a more granular level, the following table shows record discoveries broken down by match percentages of each field, for the entire dataset. This shows the extent of the matches (partially for longer query strings) and the overlap across multiple fields.

\begin{tabular}{|l|c|r|r|r|r|r|}
\hline & $\mathbf{0} \%$ & $\mathbf{1 - 4 9 \%}$ & $\mathbf{5 0 - 7 4 \%}$ & $\mathbf{7 5 - 9 9 \%}$ & $\mathbf{1 0 0 \%}$ & $\begin{array}{c}\mathbf{\%}>=\mathbf{1 \%} \\
\text { found in field }\end{array}$ \\
\hline Title & 33,597 & 2,086 & 2,297 & 120 & 5,320 & $22.62 \%$ \\
\hline Subj. & 28,661 & 1,591 & 1,736 & 61 & 11,371 & $33.99 \%$ \\
\hline Agent & 29,276 & 193 & 293 & 4 & 13,654 & $32.57 \%$ \\
\hline Descr. & 29,274 & 3,048 & 3,454 & 350 & 7,294 & $32.57 \%$ \\
\hline
\end{tabular}




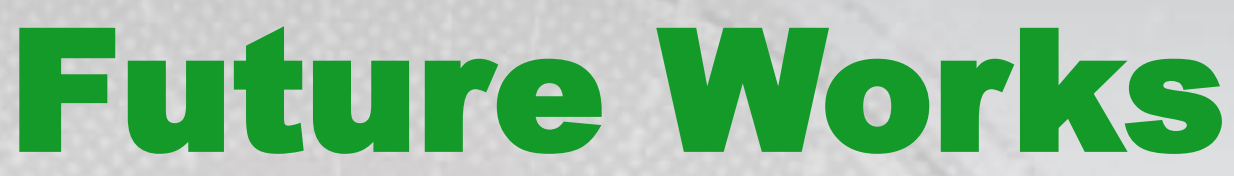

$>$ Effective metadata and taxonomies add value and amplify the mostly interdisciplinary ETDs- allowing users to explore and delve deeper in multidimensional ways.

$>$ The URLs referenced in a large corpus of ETDs may be present interesting insight into the subjects, disciplines and patterns in the ETD documents which warrants further investigation.

$>$ Additionally an investigation into how specific disciplines or subject areas are referencing URLs in their ETDs would be helpful in identifying particularly high areas of URL linking versus lower levels.

$>$ An analysis of URL inclusion in ETDs across institutions would make a logical follow-on investigation that would show if higher level trends exist in ETDs. 


\begin{tabular}{|c|c|c|c|c|c|c|c|c|c|c|c|c|c|}
\hline \multirow[t]{2}{*}{ Year } & \multirow{2}{*}{$\left|\begin{array}{c}\text { Total } \\
\text { No. } \\
\text { of } \\
\text { ETDs }\end{array}\right|$} & \multirow{2}{*}{$\begin{array}{l}\text { No. of } \\
\text { ETDs } \\
\text { with } \\
\text { URLs }\end{array}$} & \multirow{2}{*}{$\begin{array}{l}\% \text { of } \\
\text { ETDs } \\
\text { with } \\
\text { URLs }\end{array}$} & \multicolumn{2}{|c|}{ com } & \multicolumn{2}{|c|}{ org } & \multicolumn{2}{|c|}{ edu } & \multicolumn{2}{|c|}{ gov } & \multicolumn{2}{|c|}{-net } \\
\hline & & & & $\#$ & $\%$ & $\#$ & $\%$ & $\#$ & $\%$ & $\#$ & $\%$ & $\#$ & $\%$ \\
\hline 1999 & 120 & 42 & $35.00 \%$ & 14 & $42.9 \%$ & 9 & $32.1 \%$ & 11 & $39.3 \%$ & 4 & $14.3 \%$ & 4 & $14.3 \%$ \\
\hline 2000 & 315 & 127 & $40.32 \%$ & 66 & $52.0 \%$ & 70 & $55.1 \%$ & 53 & $41.7 \%$ & 41 & $32.3 \%$ & 19 & $15.0 \%$ \\
\hline 2001 & 290 & 116 & $40.00 \%$ & 63 & $54.3 \%$ & 67 & $57.8 \%$ & 57 & $49.1 \%$ & 39 & $33.6 \%$ & 20 & $17.2 \%$ \\
\hline 2002 & 298 & 146 & $48.99 \%$ & 102 & $69.9 \%$ & 73 & $50.0 \%$ & 62 & $42.5 \%$ & 47 & $32.2 \%$ & 18 & $12.3 \%$ \\
\hline 2003 & 328 & 198 & $60.37 \%$ & 133 & $67.2 \%$ & 96 & $48.5 \%$ & 84 & $42.4 \%$ & 70 & $35.4 \%$ & 24 & $12.1 \%$ \\
\hline 2004 & 304 & 181 & $59.54 \%$ & 122 & $67.4 \%$ & 89 & $49.2 \%$ & 84 & $46.4 \%$ & 66 & $36.5 \%$ & 23 & $12.7 \%$ \\
\hline 2005 & 284 & 199 & $70.07 \%$ & 141 & $70.9 \%$ & 112 & $56.3 \%$ & 100 & $50.3 \%$ & 83 & $41.7 \%$ & 43 & $21.6 \%$ \\
\hline 2006 & 326 & 235 & $72.09 \%$ & 155 & $66.0 \%$ & 143 & $60.9 \%$ & 116 & $49.4 \%$ & 98 & $41.7 \%$ & 40 & $17.0 \%$ \\
\hline 2007 & 349 & 258 & $73.93 \%$ & 182 & $70.5 \%$ & 157 & $60.9 \%$ & 122 & $47.3 \%$ & 116 & $45.0 \%$ & 35 & $16.6 \%$ \\
\hline 2008 & 336 & 242 & $72.02 \%$ & 166 & $68.6 \%$ & 140 & $57.9 \%$ & 99 & $41.0 \%$ & 91 & $37.6 \%$ & 40 & $16.5 \%$ \\
\hline 2009 & 311 & 132 & $42.44 \% *$ & 87 & $65.9 \%$ & 83 & $62.9 \%$ & 69 & $52.3 \%$ & 50 & $37.9 \%$ & 25 & $19.0 \%$ \\
\hline 2010 & 366 & 286 & $78.14 \%$ & 199 & $69.6 \%$ & 170 & $59.4 \%$ & 127 & $44.4 \%$ & 129 & $45.1 \%$ & 50 & $17.5 \%$ \\
\hline 2011 & 418 & 335 & $80.14 \%$ & 231 & $69.0 \%$ & 215 & $64.2 \%$ & 134 & $40.0 \%$ & 146 & $43.6 \%$ & 66 & $20.0 \%$ \\
\hline 2012 & 290 & 230 & $79.31 \%$ & 153 & $66.5 \%$ & 155 & $67.4 \%$ & 94 & $40.9 \%$ & 104 & $45.2 \%$ & 38 & $16.5 \%$ \\
\hline
\end{tabular}




\section{Summary}

$>$ Considering the diverse global ETDs users' communities, effective retrieval depends not only on the subject terms assigned to describe an item, but on the search query terms entered by users as well. 
management

kill ratio

hoey

economics

Distance

budget 1992

beethoven

"Eastin, Jennifer Flood"

vucca 1967

vucca 1965

prathyusha nukala

piano

physics

business administration

budget 1973

biology

vucca 1962

quintanila

miles davis

mathematics

joumalism

john coltrane

business

hobbs

child soldiers

vucca 1961

pradeep gali

history

chemistry

budget 1994

"University of North Texas"

gay

vucca 1963

psychology

biolog*

music

biopolymers making materials nature's.

vucca 1964

faculty

rose

-Human anatomy - Outlines, syllabi, etc.-

budget 1972

vahie, archna

budget 1971

education

english

budget 1970

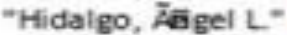

- Doctor of Musical Arts"
$7.22,74$

1 .

7.68 74

$2.85,74$

$\frac{1}{3.69} 74$

3.69

213.4274

$=10.23,74$

$4.55 \quad 87$

8.11 .55

$217.41,87$

‥23.2. 87

87.43

$9.14 .26,87$

243.87

5.5687

9.11 .03

9.99

1.51

969

5.25

2.01

917.05 .99

3.44

1.44

9.61

15.72

128

137

2.63

1.63

15.91

2.20 .74

16.89

$\begin{array}{ll}1.7 .02 & 161\end{array}$

91734

1 19.82 174

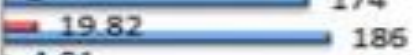

$\frac{4.01}{7.94} 223$

236

294236

3.28
1.55

1.55

259

214.712286

.752

3.56

7.09

4.8 .05
TotalUniques Searches 


\section{QUESTIONS?}

Daniel.Alemneh

@unt.edu

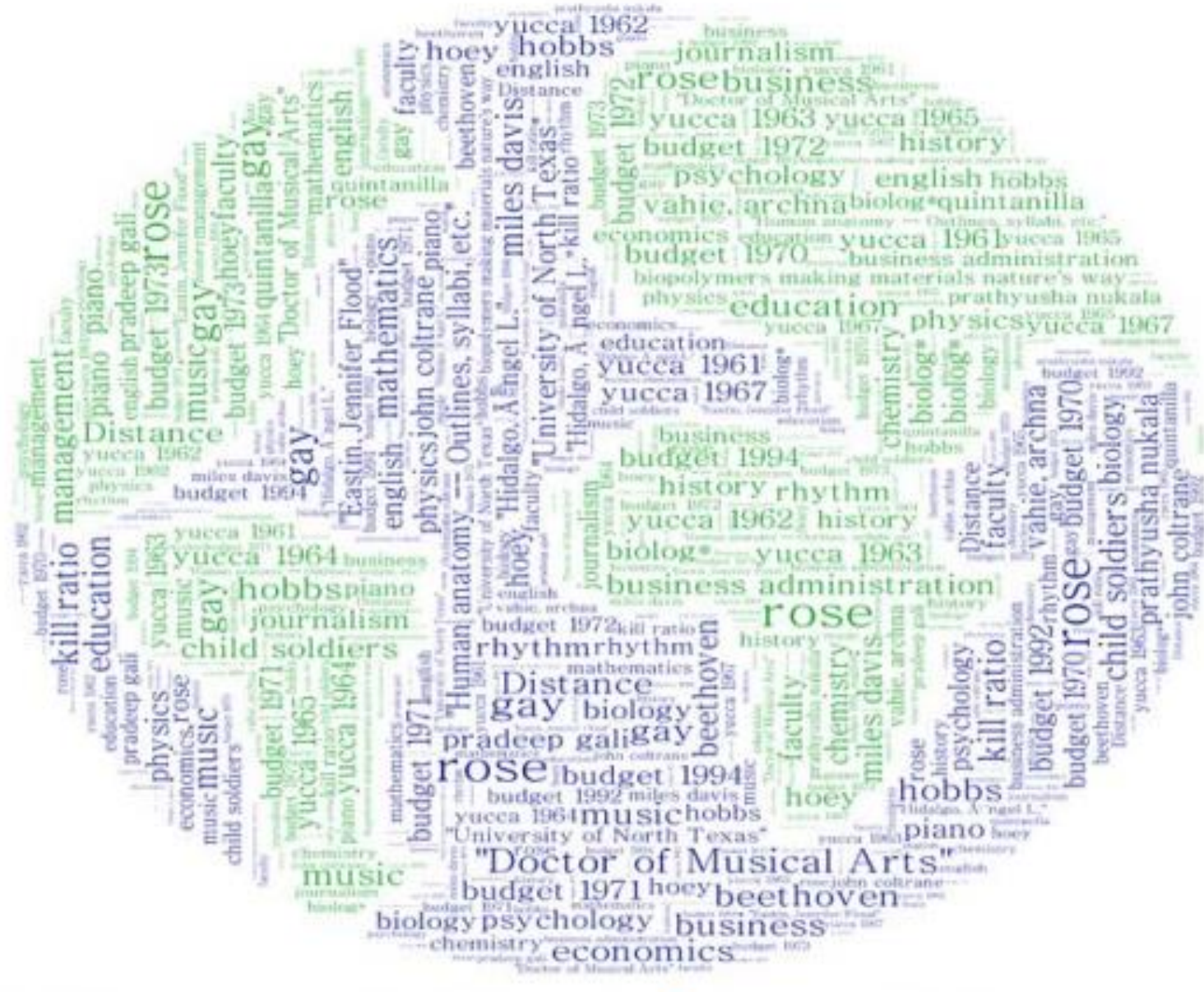

Mark.Phillips

@unt.edu 